\title{
On the Maximum Number of Equilateral Triangles, $\mathbf{I}^{*}$
}

\author{
B. M. Ábrego and S. Fernández-Merchant \\ Department of Mathematics, Rutgers University, \\ New Brunswick, NJ 08801, USA \\ \{abrego,sfernand\}@math.rutgers.edu
}

\begin{abstract}
The following problem was posed by Erdős and Purdy: "What is the maximum number of equilateral triangles determined by a set of $n$ points in $\mathbb{R}^{d}$ ?" New bounds for this problem are obtained for dimensions 2, 4, and 5. In addition it is shown that for $d=2$ the maximum is attained by subsets of the regular triangle lattice.
\end{abstract}

\section{Introduction}

In 1946 Erdôs [6] posed the seemingly innocent problem of finding the maximum number of unit segments that can be determined by a set of $n$ points in the plane. Even though the solution has long been out of reach, the quest for solving the problem generated a considerable amount of research in combinatorial geometry (see [12] for a discussion of this problem, and [2] and [15] for the latest developments).

Since then many problems sharing the same spirit have been raised (see [12]); problems concerning the maximum number of geometric objects that can be determined by an $n$-point set in a euclidean space. In this article we address one of them.

"What is the maximum number of equilateral triangles that can be determined by $n$ points in the plane?"

This problem was proposed several times by Erdős and Purdy [8]-[11], and it is also mentioned in [4].

For an arbitrary finite set $P$ denote by $E(P)$ the number of triplets in $P$ that are the vertices of an equilateral triangle, we define

$$
E(n)=\max _{\substack{|P|=n \\ P \subseteq \mathbb{R}^{2}}} E(P) .
$$

\footnotetext{
* The first author was partially supported by a DIMACS summer fellowship.
} 
It is easy to see that $E(n) \leq \frac{2}{3}\left(\begin{array}{l}n \\ 2\end{array}\right)$ since every pair of points in the plane are the vertices of at most two equilateral triangles; also by considering a suitable set of the regular triangular grid one can easily obtain $E(n)>\frac{1}{6} n^{2}$. In fact Elekes and Erdős [5] proved that for every triangle $T$, the maximum number of triangles similar to $T$ determined by $n$ points in the plane is $\Theta\left(n^{2}\right)$.

The aim of this paper is to refine these bounds. We show in Section 2 that

$$
0.1955 \leq \frac{1}{3}-\frac{\sqrt{3}}{4 \pi} \leq \liminf _{n \rightarrow \infty} \frac{E(n)}{n^{2}} \leq \frac{1}{4}=0.25,
$$

next we prove in Section 3 that $E(n)$ is achieved by subsets of the equilateral triangle lattice. Finally, in Section 4, we discuss the same problem on higher dimensions where we find lower bounds for dimensions 4 and 5, and a new upper bound for the fivedimensional space.

Throughout the paper we use the following notation. For any $P$ finite set, $G=$ $(P, \Delta(P))$ denotes the 3-uniform hypergraph with vertex set $P$, where $\Delta(P)$ is the set of triplets in $P$ determining equilateral triangles. For $x \in P$ we denote by $\operatorname{deg}_{\Delta}(x)$ the number of triangles in $\Delta(P)$ with $x$ as one of its vertices.

\section{Bounds on the Number of Triangles}

Although the order of magnitude of the function $E(n)$ is known to be quadratic, it is not even known whether $\lim _{n \rightarrow \infty}\left(E(n) / n^{2}\right)$ exists. The aim of this section is to give new bounds for $E(n)$.

For every $x \in P$ define $N_{x}=P \cap R_{x}(P) \backslash\{x\}$ where $R_{x}$ denotes the counterclockwise rotation of a $\pi / 3$ angle with center at $x$. We have that

$$
\operatorname{deg}_{\Delta}(x)=\left|N_{x}\right|=\left|P \cap R_{x}(P)\right|-1 .
$$

Theorem 1. $E(n) \leq\left\lfloor((n-1) / 2)^{2}\right\rfloor$.

Proof. It is enough to prove that any $n$-point set $P$ has a point $w$ with $\operatorname{deg}_{\Delta}(w) \leq$ $\lfloor(n-1) / 2\rfloor$. Consider points $x, y \in P$ whose distance realizes the diameter of $P$. First assume there is a point $z \in P$ such that $x y z$ is an equilateral triangle. By maximality of the segment $x y, P$ must be contained in the Reuleáux triangle $T$ with vertices $x$, $y$, and $z$. Let $X=R_{x}(T) \cap T, Y=R_{y}(T) \cap T$, and $Z=R_{z}(T) \cap T$. Observe that $N_{x} \subset X, N_{y} \subset Y, N_{z} \subset Z$ and since $x \notin N_{x}, y \notin N_{y}, z \notin N_{z}$ we have that $N_{x}, N_{y}$, and $N_{z}$ are pairwise disjoint. So

$$
\operatorname{deg}_{\Delta}(x)+\operatorname{deg}_{\Delta}(y)+\operatorname{deg}_{\Delta}(z)=\left|N_{x}\right|+\left|N_{y}\right|+\left|N_{z}\right| \leq n,
$$

hence at least one of the points $x, y$, or $z$ has $\operatorname{deg}_{\Delta} \leq\lfloor n / 3\rfloor \leq\lfloor(n-1) / 2\rfloor$.

Now suppose no point in $P$ forms an equilateral triangle together with $x$ and $y$. It follows that $y \notin N_{x}$ and $x \notin N_{y}$. We shall prove by contradiction that $\left|N_{x} \cap N_{y}\right| \leq 1$. So we assume there are two points $u$ and $v$ in $N_{x} \cap N_{y}$. This means there are points $u_{x}, v_{x}, u_{y}, v_{y} \in P$ such that all triangles $x u_{x} u, x v_{x} v, y u_{y} u, y v_{y} v$ are equilateral. Notice that the segments $u_{y} u_{x}$ and $v_{y} v_{x}$ are obtained from $y x$ by $(\pi / 3)$-counterclockwise 
rotation with centers in $u$ and $v$, respectively. So $v_{x} v_{y}$ and $u_{x} u_{y}$ are parallel and have the same length, i.e., $u_{x} v_{x} v_{y} u_{y}$ is a parallelogram. This gives us a contradiction since one of the diagonals of the parallelogram is longer than any of it sides, including those which by assumption have maximal length in $P$. Hence $\left|N_{x} \cap N_{y}\right| \leq 1$.

Therefore

$$
\left|N_{x}\right|+\left|N_{y}\right| \leq\left|N_{x} \cup N_{y}\right|+1 \leq|P \backslash\{x, y\}|+1=n-1,
$$

so either $\left|N_{x}\right| \leq\lfloor(n-1) / 2\rfloor$ or $\left|N_{y}\right| \leq\lfloor(n-1) / 2\rfloor$, i.e., one of $x$ or $y$ satisfies $\operatorname{deg}_{\Delta} \leq$ $\lfloor(n-1) / 2\rfloor$.

Denote by $\Lambda$ the lattice generated by 1 and $e^{i \pi / 3}$. The following result estimates the number of equilateral triangles determined by large clusters of points in $\Lambda$.

Theorem 2. Let $K$ be a compact set with finite perimeter and area $\sqrt{3} / 2$. Define $f_{K}: \mathbb{C} \rightarrow C$ as $f_{K}(z)=$ Area $\left(K \cap R_{z}(K)\right)$. If $K_{n}$ is a similar copy of $K$ intersecting $\Lambda$ in exactly $n$ points, then

$$
E\left(K_{n} \cap \Lambda\right)=\left(\frac{4}{9} \int_{K} f_{K}(z) d z\right) n^{2}+O\left(n^{3 / 2}\right) .
$$

Proof. Given a compact set $L$ with finite area and perimeter, we have that $|r L \cap \Lambda|=$ $(2 / \sqrt{3}) r^{2}$ Area $(L)+O(r)$ (where $r L$ denotes the magnification of $L$ by a factor $\left.r \geq 1\right)$, thus

$$
E\left(K_{n} \cap \Lambda\right)=\frac{1}{3} \sum_{x \in K_{n} \cap \Lambda}\left|N_{x}\right|=\frac{2 \sqrt{3}}{9} \sum_{x \in K_{n} \cap \Lambda}\left[\operatorname{Area}\left(K_{n} \cap R_{x}\left(K_{n}\right)\right)+O(\sqrt{n})\right] .
$$

It can be seen that all error terms are uniformly bounded, so

$$
E\left(K_{n} \cap \Lambda\right)=\frac{4 n^{2}}{9} \sum_{x \in K_{n} \cap \Lambda}\left[\frac{\sqrt{3}}{2 n} \operatorname{Area}\left(\frac{1}{\sqrt{n}} K_{n} \cap R_{x / \sqrt{n}}\left(\frac{1}{\sqrt{n}} K_{n}\right)\right)\right]+O\left(n^{3 / 2}\right) .
$$

Note that the sum above is a Riemann approximation for the function $f_{(1 / \sqrt{n}) K_{n}}$ on the region $(1 / \sqrt{n}) K_{n}$, thus

$$
E\left(K_{n} \cap \Lambda\right)=\frac{4 n^{2}}{9}\left(\int_{(1 / \sqrt{n}) K_{n}} f_{(1 / \sqrt{n}) K_{n}}(z) d z+O\left(\frac{1}{\sqrt{n}}\right)\right)+O\left(n^{3 / 2}\right),
$$

now since $\operatorname{Area}\left((1 / \sqrt{n}) K_{n}\right)=\sqrt{3} / 2+O(1 / \sqrt{n})$ then

$$
\int_{(1 / \sqrt{n}) K_{n}} f_{(1 / \sqrt{n}) K_{n}}(z) d z=\int_{K} f_{K}(z) d z+O\left(\frac{1}{\sqrt{n}}\right)
$$

and the result follows. 
The integral $\int_{K} f_{K}(z) d z$ can be precisely determined when $K$ is an equilateral triangle, a regular hexagon, or a circular disk. For these sets we obtained $\frac{1}{6}, \frac{7}{36}$, and $\frac{1}{3}-\sqrt{3} / 4 \pi \approx 0.1955$ as the corresponding quadratic coefficients of $E\left(K_{n} \cap \Lambda\right)$. Hence, together with Theorem 1, we have the following bounds for $E(n)$ :

$$
\left(\frac{1}{3}-\frac{\sqrt{3}}{4 \pi}\right) n^{2}+O\left(n^{3 / 2}\right) \leq E(n) \leq \frac{1}{4} n^{2} .
$$

Concerning the lower bound, it seems that the circular disk maximizes $\int_{K} f_{K}(z) d z$ over all compact sets $K$ with finite perimeter and area $\sqrt{3} / 2$; furthermore we conjecture that

Conjecture 1. $\lim _{n \rightarrow \infty}\left(E(n) / n^{2}\right)$ exists, and its value is $\frac{1}{3}-\sqrt{3} / 4 \pi$.

We also believe that the following stronger conjecture is true.

Conjecture 2. For any n-point set $P$, there is $x \in P$ with $\operatorname{deg}_{\Delta}(x) \leq\left(\frac{2}{3}-\sqrt{3} / 2 \pi\right)(n+$ $\sqrt{n})$.

\section{Extremal Sets}

The best evidence supporting Conjecture 1 is the next theorem, where we prove that to determine $E(n)$, it is sufficient to restrict our attention to subsets $P$ of the equilateral triangle lattice $\Lambda$.

Theorem 3. $E(n)$ is attained by subsets of the regular triangular lattice.

Proof. Consider a system of coordinates with axis forming a $\pi / 3$ angle (see Fig. 1). Take

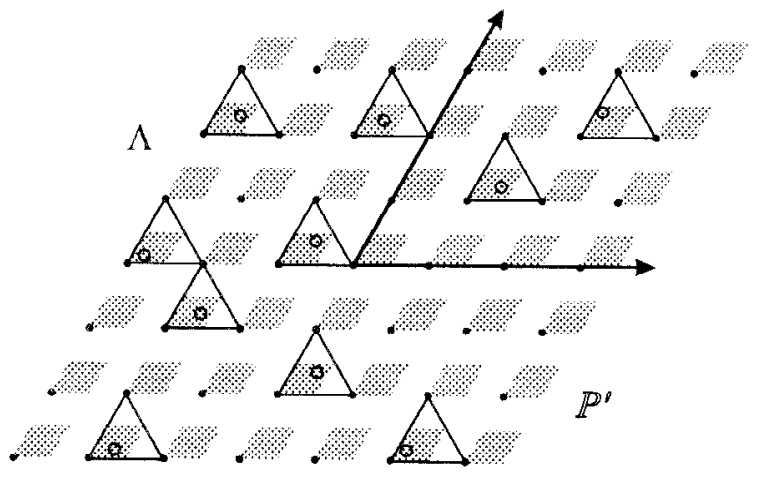

Fig. 1. $\Lambda$ and the shaded regions. 
a set $P=\left\{p_{1}, p_{2}, \ldots, p_{n}\right\}$ of $n$ points in the plane $\left(p_{j}=\left(x_{j}, y_{j}\right)\right.$ for all $\left.j=1,2, \ldots, n\right)$. Assume without loss of generality that the minimum distance between points of $P$ is $\sqrt{3} / 2$.

By the Dirichlet Simultaneous Approximation Theorem, there is an integer $1 \leq t \leq 4^{n}$ such that, for every $1 \leq j \leq n$,

$$
0<\left\{t x_{j}+\frac{1}{4}\right\}<\frac{1}{2} \quad \text { and } \quad 0<\left\{t y_{j}+\frac{1}{4}\right\}<\frac{1}{2}
$$

where $\{x\}$ denotes the fractional part of $x$.

Let $p_{j}^{\prime}=t p_{j}+\left(\frac{1}{4}, \frac{1}{4}\right)$ for $1 \leq j \leq n$, and set $P^{\prime}=\left\{p_{1}^{\prime}, p_{2}^{\prime}, \ldots, p_{n}^{\prime}\right\}$. By the choice of $t$ all points of $P^{\prime}$ are in the shaded regions indicated in Fig. 1. Moreover since any two points in $P^{\prime}$ are at a distance at least $\sqrt{3} / 2$ (by assumption on the minimum distance in $P$ ), then each connected region contains at most one point of $P^{\prime}$. In particular, all points of $P^{\prime}$ lie in the interior of a minimal triangle of $\Lambda$ pointing upward. Now consider the set $Q=\left\{q_{1}, q_{2}, \ldots, q_{n}\right\}$ of $n$ points in the plane where $q_{j}=\left(\left\lfloor t x_{j}+\right.\right.$ $\left.\left.\frac{1}{4}\right\rfloor,\left\lfloor t y_{j}+\frac{1}{4}\right\rfloor\right)$. Notice that the function $p_{j}^{\prime} \mapsto q_{j}$ is one to one, and that all points of $Q$ lie on $\Lambda$.

To conclude the proof of the theorem we verify that $E(Q) \geq E(P)$. Clearly $E(P)=$ $E\left(P^{\prime}\right)$. Now suppose that the points $p_{j}^{\prime}, p_{k}^{\prime}, p_{l}^{\prime}$ are the vertices of an equilateral triangle. Let $T_{j}$ and $T_{k}$ be the minimal triangles of $\Lambda$ to which $p_{j}^{\prime}$ and $p_{k}^{\prime}$ belong (see Fig. 2). Then the locus of all points of the plane forming an equilateral triangle with one interior point of $T_{j}$ and one interior point of $T_{k}$ is the union of the interiors of the triangles $T$ and $T^{\prime}$ in Fig. 2, where $a$ and $b$ are the unique two points forming equilateral triangles with $q_{j}$ and $q_{k}$. Since $p_{l}^{\prime}$ must be in the interior of a minimal equilateral triangle of $\Lambda$ pointing upward, then $p_{l}^{\prime}$ must lie in the interior of the shaded region indicated in Fig. 2. Therefore either $q_{l}=a$ or $q_{l}=b$, which means that the points $q_{j}, q_{k}, q_{l}$ are the vertices of an equilateral triangle, and hence $E(Q) \geq E\left(P^{\prime}\right)$.

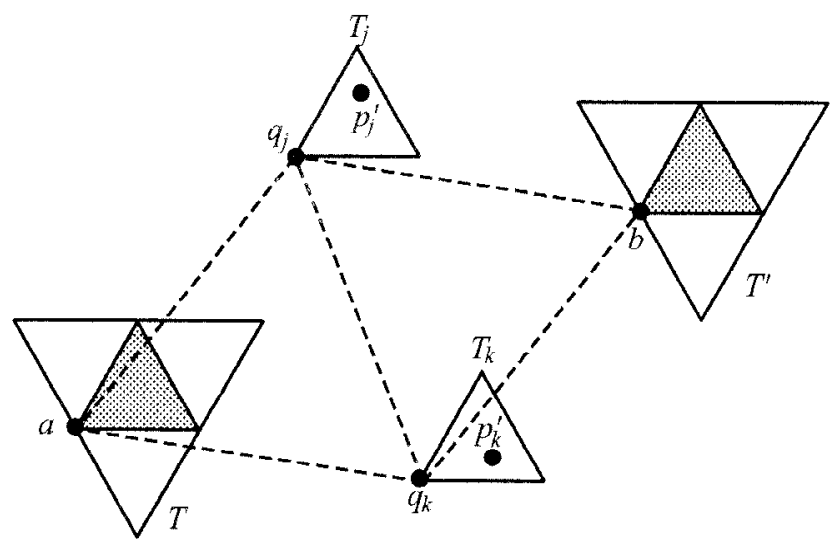

Fig. 2. Illustration for the proof of Theorem 3. 


\section{Higher Dimensions}

For $d \geq 2$ let $E_{d}(n)$ denote the maximum number of equilateral triangles determined by $n$ points in $\mathbb{R}^{d}$. Similarly define $F_{d}(n)$ where the maximum is restricted to the number of unit equilateral triangles.

A generalized Lenz's construction [8] shows that $n^{3}$ is actually the correct order of magnitude of both functions $E_{d}(n)$ and $F_{d}(n)$ when $d \geq 6$. Regarding the unitary case for $d=2$ the best results are $n^{1+c / \log \log n} \leq F_{2}(n) \leq O\left(n^{4 / 3}\right)$ (both bounds follow from the unitary distance problem [6], [14]). For $d=3,4$, the upper bounds $E_{3}(n)=O\left(n^{2.2}\right), F_{3}(n)=O\left(n^{1.8+\varepsilon}\right)$, and $F_{4}(n)=O\left(n^{65 / 23}\right)$ were proven by Akutsu et al. [1]. Purdy [13] proved as a special case of a more general result that $E_{4}(n)=O\left(n^{2.96}\right)$.

Now we give an upper bound for $E_{5}(n)$ which in turn improves the bound above for $E_{4}(n)$.

Theorem 4. $\quad E_{5}(n)=O\left(n^{3-1 / 9}\right)$.

Proof. Suppose that for a sufficiently large $n$ there is an $n$-point set $P$ determining at least $n^{3-1 / 9}$ equilateral triangles. Consider the 3-uniform hypergraph $G=(P, \Delta(P))$. By Erdôs's theorem [7], $G$ contains a subhypergraph $H$ isomorphic to $K_{3}^{(3)}$ (the 3uniform hypergraph consisting of nine vertices partitioned into three equal size classes, and whose set of 3-edges is formed by all triangles with exactly one vertex in each class).

Assume $x_{1}, x_{2}, x_{3}, y_{1}, y_{2}, y_{3}, z_{1}, z_{2}, z_{3}$ are the vertices of $H$ and all triplets of the form $\left\{x_{j}, y_{k}, z_{l}\right\}$ are the corresponding 3 -edges. Assume without loss of generality that all sides of triangles in $H$ have unit length.

Then for all $j \in\{1,2,3\}$ the set $\left\{y_{1}, y_{2}, y_{3}, z_{1}, z_{2}, z_{3}\right\}$ is contained in the fourdimensional unit sphere $S_{x_{j}}$ with center in $x_{j}$. This implies that the three-dimensional spheres $S_{1,2}:=S_{x_{1}} \cap S_{x_{2}}$ and $S_{1,3}:=S_{x_{1}} \cap S_{x_{3}}$ also contain the set $\left\{y_{1}, y_{2}, y_{3}, z_{1}, z_{2}, z_{3}\right\}$. Note that the spheres $S_{1,2}$ and $S_{1,3}$ are distinct, since their centers are the middle points of the segments $x_{1} x_{2}$ and $x_{1} x_{3}$, respectively. Hence $S:=S_{1,2} \cap S_{1,3}$ is a two-dimensional sphere containing $\left\{y_{1}, y_{2}, y_{3}, z_{1}, z_{2}, z_{3}\right\}$. Let $V_{1,2}$ be the set of points in $S$ equidistant to $y_{1}$ and $y_{2}$, and similarly define $V_{1,3}$. Then $V_{1,2}$ and $V_{1,3}$ are distinct circles contained in $S$. To get a contradiction observe that $\left\{z_{1}, z_{2}, z_{3}\right\} \subseteq V_{1,2} \cap V_{1,3}$ but $\left|V_{1,2} \cap V_{1,3}\right| \leq 2$.

To complete this section we present examples in dimensions 4 and 5 with a large number of unit equilateral triangles.

Theorem 5. $F_{4}(n)=\Omega\left(n^{2}\right)$ and $F_{5}(n)=\Omega\left(n^{7 / 3}\right)$.

Proof. Let $A \subseteq \mathbb{R}^{2}$ be the set of vertices of $\lfloor n / 8\rfloor$ unit squares centered at the origin. Define $P \subseteq \mathbb{R}^{2} \times \mathbb{R}^{2}$ by $P=(A \times\{0\}) \cup(\{0\} \times A)$. Notice that if $a_{1} a_{2}$ is a unit segment in $A$, then the triangle $(a, 0),\left(0, a_{1}\right),\left(0, a_{2}\right)$ is equilateral for any $a \in A$. Therefore

$$
F_{4}(n) \geq F(P) \geq 32\left\lfloor\frac{n}{8}\right\rfloor^{2}=\Omega\left(n^{2}\right) .
$$


For the second part consider the sphere $S \subseteq \mathbb{R}^{3}$ of radius $1 / \sqrt{2}$ centered at the origin. Clarkson et al. [3] proved the existence of an $\lfloor n / 2\rfloor$-point set $B \subseteq S$ which determines $\Omega\left(n^{4 / 3}\right)$ unit distances. Define the set $Q=(A \times\{0\}) \cup(\{0\} \times B) \subseteq \mathbb{R}^{2} \times \mathbb{R}^{3}$. Again if $b_{1} b_{2}$ is a unit segment in $B$, then the triangle $(a, 0),\left(0, b_{1}\right),\left(0, b_{2}\right)$ is equilateral for any $a \in A$. Therefore

$$
F_{5}(n) \geq F(Q) \geq n \cdot \Omega\left(n^{4 / 3}\right)=\Omega\left(n^{7 / 3}\right) .
$$

\section{References}

1. T. Akutsu, H. Tamaki, and T. Tokuyama. Distribution of Distances and Triangles in a Point Set and Algorithms for Computing the Largest Common Point Sets. Discrete \& Computational Geometry 20 (1998), 307-331.

2. P. Brass. On Point Sets with Many Unit Distances in Few Directions. Discrete \& Computational Geometry 19 (1998), 355-366.

3. K. L. Clarkson, H. Edelsbrunner, L. J. Guibas, M. Sharir, and E. Welzl. Combinatorial Complexity Bounds for Arrangements of Curves and Spheres. Discrete \& Computational Geometry 5 (1990), 99-160.

4. H. T. Croft, K. J. Falconer, and R. K. Guy. Unsolved Problems in Geometry. Springer-Verlag, New York, 1991.

5. G. Elekes and P. Erdős. Similar Configurations and Pseudogrids. In Intuitive Geometry. Colloquia Mathematica Societatis János Bolyai. North-Holland, Amsterdam, 1994, pp. 85-104.

6. P. Erdôs. On Sets of Distances of $n$ Points. American Mathematical Monthly 53 (1946), 248-250.

7. P. Erdős. On Some Problems of Graphs and Generalized Graphs. Israel Journal of Mathematics 2 (1964), 183-190.

8. P. Erdős. On Some Problems of Elementary and Combinatorial Geometry. Annali di Matematica Pura Applicata Serie Quarta 103 (1975), 99-108.

9. P. Erdős and G. Purdy. Some Extremal Problems in Geometry, Journal of Combinatorial Theory 10 (1971), 246-252.

10. P. Erdős and G. Purdy. Some Extremal Problems in Geometry, III. Proc. 6th Southeastern Conference on Combinatorics, Graph Theory and Computation, 1975, pp. 291-308.

11. P. Erdős and G. Purdy. Some Extremal Problems in Geometry, IV. Proc. 7th Southeastern Conference on Combinatorics, Graph Theory and Computation, 1976, pp. 307-322.

12. J. Pach and P. K. Agarwal. Combinatorial Geometry, Wiley, New York, 1995.

13. G. Purdy. Repeated Angles in $E^{4}$. Discrete and Computational Geometry 3 (1988), 73-75.

14. J. Spencer, E. Szemerédi, and W. Trotter, Jr. Unit Distances in the Euclidean Plane. In Graph Theory and Combinatorics (B. Bollobás, ed.). Academic Press, New York, 1984, pp. 293-303.

15. L. A Szekely. Crossing Numbers and Hard Erdôs Problems in Discrete Geometry. Combinatorics, Probability \& Computing 7 (1997), 353-358.

Received April 9, 1998, and in revised form October 30, 1998. 